\section{Rates of Adherence to Hand Hygiene and Gloving Practices in 2 French Rehabilitation Hospitals by Differentiation between Single Contacts and Series of Successive Contacts with Patients or the Environment}

To the Editor-Although adherence to hand hygiene is considered to be the cornerstone of the prevention of pathogen cross-transmission,' the overall rate of adherence remains low-less than $50 \%$ in most hospitals. ${ }^{2-4}$ Concurrently, glove use has been shown to modify adherence to hand hygiene and to modify the perception of hand hygiene. ${ }^{5}$ Although numerous studies concerning hand hygiene adherence have been performed in various settings, few data exist about either hand hygiene or gloving practices in rehabilitation units. We monitored the rates of adherence to hand hygiene and the gloving practices in 2 rehabilitation settings to assess adherence to recommendations.

A direct observational prospective study was conducted for 2 weeks in October 2009. This study was performed in 2 rehabilitation hospitals, one located in Angers (western France) and the other in the area surrounding Angers.

Opportunities for hand hygiene were defined in accordance with the leaflet by the World Health Organization titled "My Five Moments for Hand Hygiene." Hand hygiene was considered to occur if hand washing or hand rubbing with an alcohol-based product was performed. Contacts were differentiated into those with risk of exposure to body fluid and those without such risk. In addition, opportunities for hand hygiene were also differentiated as extraseries opportunities (before or after a single contact, or before the first contact and after the last contact of a series of successive contacts) or as intraseries opportunities (from the opportunity after the first contact to the opportunity preceding the last contact of a series of successive contacts). For a single contact, 2 opportunities for hand hygiene ( 1 before and 1 after contact) were possible, whereas only 1 opportunity was possible between 2 successive contacts. Practices of glove use were evaluated by the 3 following indicators: the percentage of gloves worn as indicated, calculated as follows: [number of contacts with glove use and risk of exposure to body fluids/number of contacts with glove use] $\times 100$; the percentage of gloves worn if indicated, calculated as follows: [number of contacts with glove use and risk of exposure to body fluids/number of contacts with risk of exposure to body fluids] $\times 100$; and the percentage of glove misuse, calculated as follows: [ number of contacts with glove use but no indication for glove use plus the number of contacts with risk of exposure to body fluids but without glove use) divided by the total number of contacts] $\times 100$.

Data acquisition and analysis were performed with EpiInfo software, version 6.04 (Centers for Disease Control and Prevention). Rates of adherence were expressed with $95 \%$ confidence intervals.

Overall, 304 contacts were observed $(30.6 \%$ of them were single contacts, whereas $69.4 \%$ were included in a series of successive contacts), representing 456 opportunities for hand hygiene. The percentage of all contacts that involved risk of exposure to body fluids was $17.1 \%$. Results of the different indicators used to assess hand hygiene and gloving practices are presented in Table 1. It is noteworthy that 68 opportunities concerned physiotherapists. For those opportunities, the adherence rate among intraseries opportunities was $34.6 \%$ and the adherence rate among extraseries opportunities was $78.6 \%$. Eighty-seven opportunities were observed after hours (from 6:00 $\mathrm{PM}$ to 10:00 $\mathrm{PM}$ ), showing an adherence rate of $47.8 \%$ for intraseries opportunities and of $95.3 \%$ for extraseries opportunities. The adherence rate for extraseries opportunities was substantially higher after hours than before $6: 00$ PM $(95.3 \%$ vs $81.3 \% ; P<.01)$. Finally, gloves were worn for 126 contacts ( $41.4 \%$ of all observed contacts).

Two studies entirely or partially conducted in rehabilitation units reported adherence rates for hand hygiene. According to Girou et $\mathrm{al}^{7}{ }^{7}$ the adherence rate measured in 5 units of a French rehabilitation hospital was $60.8 \%$, whereas another study performed in a 28 -bed rehabilitation unit in Italy reported an adherence rate nearly 4 -fold lower $(15.8 \%) .^{3}$ Our results are closer to those of the former study. However, because extraseries opportunities and intraseries opportunities have not been differentiated in preceding studies, it is not easy to compare their adherence rates with those of our study. Indeed, it has already been demonstrated that the proportion of all the observed opportunities that represent intraseries

TABLE 1. Rates of Adherence to Hand Hygiene and Gloving Practices in 2 Rehabilitation Hospitals in France

\begin{tabular}{lcc}
\hline Variable & $\begin{array}{c}\text { No. of } \\
\text { observations }\end{array}$ & $\begin{array}{c}\text { Rate of adherence, } \\
\%(95 \% \mathrm{CI})\end{array}$ \\
\hline $\begin{array}{l}\text { Hand hygiene opportunities } \\
\quad \text { Intraseries }\end{array}$ & 152 & $44.1(36.2-52.0)$ \\
$\quad$ Extraseries & 304 & $84.2(80.1-88.3)$ \\
$\quad$ Overall & 456 & $70.8(66.6-75.0)$ \\
Hand hygiene after gloving & 126 & $61.9(53.4-70.4)$ \\
Glove use if indicated & 78 & $78.2(69.0-87.2)$ \\
Glove use as indicated & 126 & $48.4(39.7-57.1)$ \\
Glove misuse & 302 & $27.2(22.2-32.2)$ \\
\hline
\end{tabular}

NOTE. CI, confidence interval.

a Absence of glove use when indicated or presence of glove use when not indicated. 
opportunities influences greatly the calculated overall rate of adherence. $^{\text {? }}$

The high adherence rate reported for extraseries opportunities in our study is consistent with a high level of prevention of transmission of microorganisms from patients to others. An association between the rate of adherence to hand hygiene and the prevalence of methicillin-resistant Staphylococcus aureus has already been demonstrated in a French rehabilitation hospital. ${ }^{6}$ Conversely, because of the low rate of adherence to hand hygiene in intraseries opportunities in our study, there was a substantial risk of infection that corresponded to the potential transmission of organisms from colonized sites to sterile sites within the same patient.

In our study, rates of adherence to hand hygiene and gloving practices were closely related. The rate of adherence to hand hygiene after contact with gloves (61.9\%) was higher than the corresponding rate of adherence recorded in medical wards $(44.6 \%)$ and in intensive care units $(53.3 \%)$ by Girou et al. ${ }^{5}$ The percentage of contacts with risk of exposure to body fluid but without glove use (ie, glove use indicated but not performed; $21.8 \%$ ) reveals a marked risk of contamination of healthcare workers with pathogens from blood or other body fluids and was higher than the corresponding percentage in the study by Girou et $\mathrm{al}^{5}(14.0 \%$ in medical wards and $6.4 \%$ in intensive care units).

Implementing an intervention program could help increase rates of adherence to hand hygiene among intraseries opportunities. For example, the introduction of a patient education model in an inpatient rehabilitation unit of an acute care university hospital in Pennsylvania raised the frequency of hand hygiene from 5 episodes per patient-day to 9 episodes per patient-day during the intervention and to 7 episodes per patient-day 3 months after the intervention. ${ }^{8}$

\section{ACKNOWLEDGMENTS}

Potential conflicts of interest. All authors report no conflicts of interest relevant to this article.

Matthieu Eveillard, PharmD, PhD;

Armelle Rabjeau, PharmD; Marie-Thérèse Pradelle, RN; Françoise Raymond, RN;

Marie-Laure Joly-Guillou, MD, PhD; Patrick Brunel, MD

From Laboratoire de Bactériologie-Hygiène, Centre Hospitalier Universitaire d'Angers (M.E., M-L.J-G.), Réseau ANJELIN, Centre Régional de Rééducation et Réadaptation Fonctionnelles (A.R., M-T.P., P.B.), Angers, and Antenne Régionale de Lutte contre les Infections Nosocomiales, Nantes (F.R.), France.

Address reprint requests to Matthieu Eveillard, PharmD, $\mathrm{PhD}$, Centre Hospitalier Universitaire d'Angers, 4 Rue Larrey, Angers, F-49000, France (MaEveillard@chu-angers.fr).

Infect Control Hosp Epidemiol 2010; 31(8):878-879

(C) 2010 by The Society for Healthcare Epidemiology of America. All rights reserved. 0899-823X/2010/3108-0023\$15.00. DOI: $10.1086 / 655436$

\section{REFERENCES}

1. Pittet D. Compliance with hand disinfection and its impact on hospitalacquired infections. $J$ Hosp Infect 2001;48(suppl A):540-546.

2. Pittet D, Mourouga P, Perneger TV. Compliance with handwashing in a teaching hospital. Ann Intern Med 1999;130:126-130.

3. Pan A, Domenighini F, Signorini $L$, et al. Adherence to hand hygiene in an Italian long-term care facility. Am J Infect Control 2008;36:495-497.

4. Saint S, Bartoloni A, Virgili G, et al. Marked variability in adherence to hand hygiene: a 5-unit observational study in Tuscany. Am J Infect Control 2009;37:306-310.

5. Girou E, Chai SHT, Oppein F, et al. Misuse of gloves: the foundation for poor compliance with hand hygiene and potential for microbial transmission. J Hosp Infect 2004;57:162-169.

6. Sax H, Allegranzi B, Uçkay I, Larson E, Boyce J, Pittet D. "My five moments for hand hygiene": a user-centred design approach to understand, train, monitor, and report hand hygiene. J Hosp Infect 2007;67:9-21.

7. Girou E, Legrand P, Soing-Altrach S, et al. Association between hand hygiene compliance and methicillin-resistant Staphylococcus aureus prevalence in a French rehabilitation hospital. Infect Control Hosp Epidemiol 2006;27:1128-1130.

8. McGuckin M, Taylor A, Martin V, Porten L, Salcido R. Evaluation of a patient education model for increasing hand hygiene compliance in an inpatient rehabilitation unit. Am J Infect Control 2004;32:235-238.

\section{Prevention of Infection Caused by Gram- Positive Bacteria in the Bloodstream and Lungs}

To the Editor-We read with interest the article by MunozPrice et $\mathrm{al}^{1}$ concerning a reduction in cases of bloodstream infection that was associated with the daily use of chlorhexidine baths for patients at a long-term acute care hospital in the greater Chicago area. We have a long-term acute care hospital in Tampa, Florida, with a similar size and patient mix as the one in the study by Munoz-Price et al. ${ }^{1}$ In 1995, we published an article ${ }^{2}$ showing a significant reduction in cases of methicillin-resistant Staphylococcus aureus (MRSA) ventilator-associated pneumonia (VAP) that was associated with the use of an infection prevention protocol similar to the one described in the study by Munoz-Price et al. ${ }^{1}$ We gave our patients twice-weekly chlorhexidine baths and applied a topical mupiracin ointment to their anterior nares. After the use of this protocol, we found a similar decrease in the bloodstream infection rate: from more than 10 cases per 1,000 ventilator-days to less than 2 cases per 1,000 ventilatordays. To date, this rate has been maintained because we have continued to use our infection prevention protocol. We suggest that a study comparing the use of twice-weekly chlorhexidine baths and topical mupirocin ointment to the anterior nares of patients with the use of daily chlorhexidine baths be performed to determine whether daily washes are necessary and whether the rate of MRSA VAP is reduced. Daily washes with chlorhexidine are expensive and a lot of 